Original article

\title{
Intake of energy providing liquids during the first year of life in five European countries
}

\author{
Sonia A. Schiess ${ }^{a}$, Veit Grote ${ }^{b}$, Silvia Scaglioni ${ }^{c}$, Veronica Luque ${ }^{d}$, Francoise Martin ${ }^{e}$, \\ Anna Stolarczyk ${ }^{\mathrm{f}}$, Fiammetta Vecchi ${ }^{\mathrm{c}}$, Berthold Koletzko ${ }^{\mathrm{a}, *}$ for the European Childhood \\ Obesity Project ${ }^{\mathrm{g}}$ \\ ${ }^{a}$ Div. Metabolic and Nutritional Medicine, Dr. von Hauner Children's Hospital, University of Munich Medical Centre, Lindwurmstr. 4, D-80337 Munich, Germany \\ ${ }^{\mathrm{b}}$ Dept. Epidemiology, Inst. of Social Paediatrics and Adolescent Medicine, University of Munich, Germany \\ ${ }^{\mathrm{c}}$ Dept. of Paediatrics, San Paolo Hospital, University of Milan, Italy \\ ${ }^{\mathrm{d}}$ Paediatrics Research Unit, University Rovira $i$ Virgili, Reus, Spain \\ e CHC St. Vincent, Rocourt, Belgium \\ ${ }^{\mathrm{f}}$ Clinic of Paediatrics, Children's Memorial Health Institute, Warsaw, Poland
}

\section{A R T I C L E I N F O}

\section{Article history:}

Received 22 July 2009

Accepted 20 April 2010

\section{Keywords:}

Energy providing liquids

Instant tea

Fruit juice

Complementary feeding

3-day weighed food protocol

Infants

\begin{abstract}
S U M M A R Y
Background: Intake of energy providing liquids (EPL) other than breast milk or formula to infants is discouraged because it may displace milk intake. Data on actual practice is lacking.

Aim: To describe the current practice of EPL supply to infants in five European countries.

Method: Breastfed (BF) infants and infants fed using two formulae (FF) with different protein content were recruited from October 2002 to June 2004. Three-day weighed food protocols of 1368 infants were obtained monthly at the ages of 1 to 9 and again at 12 completed months.

Results: At the age of 4 months, $13 \%$ of $\mathrm{BF}$ and $43 \%$ of FF infants received EPL. FF infants started EPL earlier (median 17 weeks) than BF infants (median 30 weeks). EPL intake was associated with a lower intake of formula milk and solids ( $\mathrm{kcal} / \mathrm{d}$ ) in the first year of life. Multiple regression analysis showed significant differences in EPL introduction between the individual countries.

Conclusion: In contrast to recommendations, EPL is frequently given during the first months of life to breastfed and particularly to formula fed infants. Infants given EPL showed lower intakes of infant formula and solids. Caregivers should receive better counselling on appropriate infant feeding.
\end{abstract}

(c) 2010 Elsevier Ltd and European Society for Clinical Nutrition and Metabolism. All rights reserved.

\section{Introduction}

Healthy infants should preferably be breastfed and should not receive complementary foods (solids and liquids other than breast

Abbreviations: AAP, American Academy of Paediatrics; EPL, energy providing liquids; BF, breast fed; FF, formula fed; ESPGHAN, European Society for Paediatric Gastroenterology, Hepatology and Nutrition.

* Corresponding author. Tel.: +49 895160 2826; fax: +49 8951607742.

E-mail address: office.koletzko@med.uni-muenchen.de (B. Koletzko).

g Study team: Belgium (ULB Bruxelles and $\mathrm{CHC}$ St Vincent Liège): Carlier $\mathrm{C}$ Goyens P, Hoyos J, Langhendries J-P, Martin F, Van Hees J-N, Xhonneux A; Germany (Division of Paediatric Epidemiology, Institute of Social Pediatrics and Adolescent Medicine and Division of Nutritional Medicine and Metabolism, Dr. von Hauner Children's Hospital, Ludwig Maximilians University of Munich): Beyer J, Demmelmair H, Fritsch M, Handel U, Hannibal I, Kreichauf S, von Kries R, Pawellek I, Verwied-Jorky S; Italy (University of Milan): Giovannini M, Agostoni C, Confalonieri F, Scaglioni S, Tedeschi S, Vecchi F, Verduci E; Spain (Universitat Rovira i Virgili): Closa Monasterolo R, Escribano Subias J, Méndez Riera G, Luque Moreno V; Poland (Children's Memorial Health Institute): Socha J, Dobrzańska A, Gruszfeld D, Socha P, Stolarczyk A, Janas R, Pietraszek E, Kowalik A. milk, infant formula or follow-on formula) in addition to breastfeeding (or formula feeding) before 17 weeks nor later than 26 weeks of age. ${ }^{1,2}$ Infants do not require additional liquids like water, tea, juices, sweetened beverages or other energy providing liquids (EPL) other than breast milk or infant formula during at least the first half year of life, with the possible exception of selected conditions such as diarrhoea, very high ambient temperatures, high fever or other excessive fluid losses, or in some selected indications occurring in the neonatal period. ${ }^{3}$

The provision of EPL during the first year of life is not recommended. Feeding infants with EPL may displace breast milk or infant formula intake and, thereby, may adversely affect nutrient supply. ${ }^{4}$ Moreover, infants with regular intake of EPL might be primed to their sweet taste with a possible increased risk for later development of dental caries or obesity. ${ }^{4-7}$ High intakes of fruit juice can exceed the capacity for fructose absorption and induce diarrhoea, abdominal pain and growth faltering. ${ }^{8-10,4}$ 
The American Academy of Paediatrics (AAP) concluded that fruit juices have no nutritional advantages over whole fruit; they lack fibre, are consumed more quickly and do not promote the desirable behaviour of eating whole fruits. ${ }^{4}$ The AAP recommended to provide fruit juices only after the age of 6 months when infants can drink from a cup, and only as part of a meal in order to reduce the risk of dental caries induction, ${ }^{4}$ whereas others recommended not providing fruit juice before the age of 9 months. ${ }^{11}$ In contrast to these recommendations, an increasing use of fruit juices in the infant diet has been reported. ${ }^{12}$ Sales of juices for infants in the United States increased from $9.7 \%$ of total complementary feeding products in 1971 to $16.7 \%$ in 1984 and probably further by $1992 .{ }^{12}$

There is a lack of current data on EPL consumption by infants. We aimed to characterize the practice of introducing EPL to infants in five European countries with similar infant feeding recommendations. Data was collected as part of the prospective European Childhood Obesity Project. We explored whether type of milk feeding, socio-demographic characteristics and the country of residence were associated with EPL use, and whether EPL consumption influences the intake of infant formula or solids.

\section{Subjects and methods}

\subsection{Study design}

Data were collected as part of the European Childhood Obesity Project, a double-blind, randomized controlled trial with one group of breastfed (BF) infants and two groups of formula fed (FF) infants randomized to formula with different protein levels as a possible risk factor for later obesity. The methodology of the study has been previously reported. ${ }^{13,14}$ In short, eligible participants were healthy, singletons, term infants recruited between 1 October 2002 to 31 June 2004 and followed-up in 11 study centres in 5 European countries (Belgium, Germany, Italy, Poland and Spain). Mothers were contacted at maternity hospitals by trained study personnel, or after hospital discharge by midwives or paediatricians.

After receiving oral and written information of the study and providing written consent, parents were invited to the first medical visit for collection of socioeconomic data, medical history of parents and infant, and infant anthropometry. During the following months, parents and infants were followed at regular intervals in the study centres, as well as by mailed questionnaires on feeding behaviours.

\subsection{Dietary intervention}

A reference group of infants fully breastfed for at least 3 months (BF) was followed without any intervention. Formula fed infants (FF) were randomized not later than 8 weeks of age to receive two infant formulae and later follow-on formulae with either higher or lower protein levels for the duration of the first year of life. ${ }^{14}$ There was no other intervention with respect to the infant diet.

\subsection{Data collection}

All data other than food protocols were collected by standardized questionnaires. A 3-day weighed food protocol was chosen to collect detailed information on the infant food and nutrient intake. Parents were asked to complete weighed food protocol on 3 consecutive days, including 2 week days and one weekend day, monthly at the ages of 1 to 9 completed months and again at 12 months. Volumes of milk were recorded based on the scale in the feeding bottles. Other food items were weighed on a digital food scale with an accuracy of $1 \mathrm{~g}$ (Soehnle unica, No. 66006, Nassau, Germany) given to the parents at the 3-month study visit. Parents were asked to record the time and place of feeding, the intake of all milk, liquid and solid food; for FF infants, the quantity (grams) of water, milk powder or cereals used for the preparation of each formula bottle, the amount ( $\mathrm{ml}$ ) of formula milk offered and the amount actually consumed by the infant. Parents were requested to note any intake of liquid other than breast or infant formula, the product and brand name of the liquid, and the time when it was first introduced. All other food items, their brand names or the recipe in case of home prepared foods, the quantity (grams) of food offered and the quantity consumed by the infant, were recorded. Trained dieticians in all study centres entered the data of the food protocols from their centre using a special software developed for this study. Nutrient content data of foods was derived from the German Food Code and Nutrient data base Bundeslebensmittel schlüssel, BLS II.3 (Federal Institute for Risk Assessment, Berlin, Germany). Nutrient contents of new food items as well as country specific foods were added to the data base as required. Standard Operating Procedures (SOPS) were developed and implemented, and dietary study staff participated in semi-annual training workshops to ensure consistent procedures and quality for data introduction and calculations in all participating centres.

We were unable to measure breast milk volume intake in the study population of the 5 countries under the conditions of the study.

The two infant formula groups with different protein levels did not differ in the intake of EPL, fruit juice, vegetable juice or instant tea. Therefore, we choose to describe the results on EPL intake for the combined group of all formula fed infants.

\subsection{Definition of energy providing liquids (EPL)}

Complementary foods (3281 food items) consumed during the first year of life by the infants in the 5 countries were classified by their ingredients and categorized into subgroups. For this analysis, EPL were defined as sugared instant tea, fruit juices (100\% fruit juice, fruit drinks), vegetable juices provided as drinks (but not as one ingredient of a composed dish), and other sugared beverages (soft drinks, sugared water without or with flavours).

\subsection{Socio-demographic data}

Mothers were categorized into three education levels (low $=$ preliminary to lower secondary, middle $=$ upper secondary and post-secondary, and high $=$ first and second stage of tertiary education) and by age at birth ( $\mathrm{I}=\leq 25, \mathrm{II}=>25$ to 30 , III $=>30$ to 35 and $\mathrm{IV}=>35$ years).

\subsection{Statistics}

All analyses were stratified by feeding type (breastfed/formula fed). Chi-square test and multiple logistic regression were used to analyse the time points of EPL introduction. We applied multiple logistic regression models for each month to detect associations between maternal age, education level, smoking behaviour, country of residence and times of introducing EPL. Wilcoxon ranksum tests were performed to compare the energy intakes of infants with and without EPL consumption. Stata 9.2, SPSS 16.0 and Excel 2000 were used for data analysis.

\subsection{Ethics}

The study protocol was reviewed and accepted by the ethic committees at all study centres.

\section{Results}

At least one informative 3-day weighed food protocol was available for 1368 infants ( $82 \%$ of the 1678 infants recruited). Of the 
1184 children with food protocols still participating in the study at 6 months, 875 (74\%) completed all 6 monthly food protocols until this age.

\section{BF infants}

Some $75 \%$ of BF infants received EPL during the first year of life. The median age at introduction was 30 weeks. Ten percent of breastfed infants received EPL at 9 weeks, $8 \%$ to $10 \%$ of the $\mathrm{BF}$ infants received EPL (mostly instant tea) during the first 3 months of life (Fig. 1A). From 4 to 6 completed months, the proportion of BF infants introduced to EPL increased from $13 \%$ to $36 \%$.

There were marked differences in EPL consumption between countries (Fig. 2A). At the age of 1 month, $8 \%$ of BF infants in Italy, $14 \%$ in Poland and 10\% in Spain, received EPL. At 3 months we found the highest proportions of infants with EPL intake in Poland and Italy and at 4 to 9 months in Poland and Spain.

Also the type of EPL consumed differed considerably between the countries. Not more than $10 \%$ of the BF infants in Germany,
Belgium and Spain consumed instant tea during the first year of life, whereas 56 \% of BF infants in Poland and 29\% in Italy received instant tea (Table S1).

At the age of 4 completed months $7 \%$ of BF infants received fruit juice. Early introduction (mainly orange juice) was seen especially in Spain, where 23\% of all infants received some at the age of 4 completed months (Table S1). The highest proportions of BF infants with an intake of fruit juice were found in the second half of the year in Poland (34\% to 66\%) and Spain (44\% to 61\%), and at the end of the first year in Germany (58\%), whereas Italian infants showed the lowest proportion (13\% to 29\%) (data not shown). Vegetable juice (mostly carrot juice) was consumed almost exclusively by Polish and German BF infants (Table S1). No BF infants had received vegetable juice until the age of 4 months while $2 \%$ of $B F$ infants in Belgium, $7 \%$ in Germany and $16 \%$ in Poland consumed some vegetable juice at the age of 6 months.

Beverages like soft drinks or sugar-added flavoured waters were hardly consumed at all by BF infants $(<0.5 \%)$ during the first year of life.
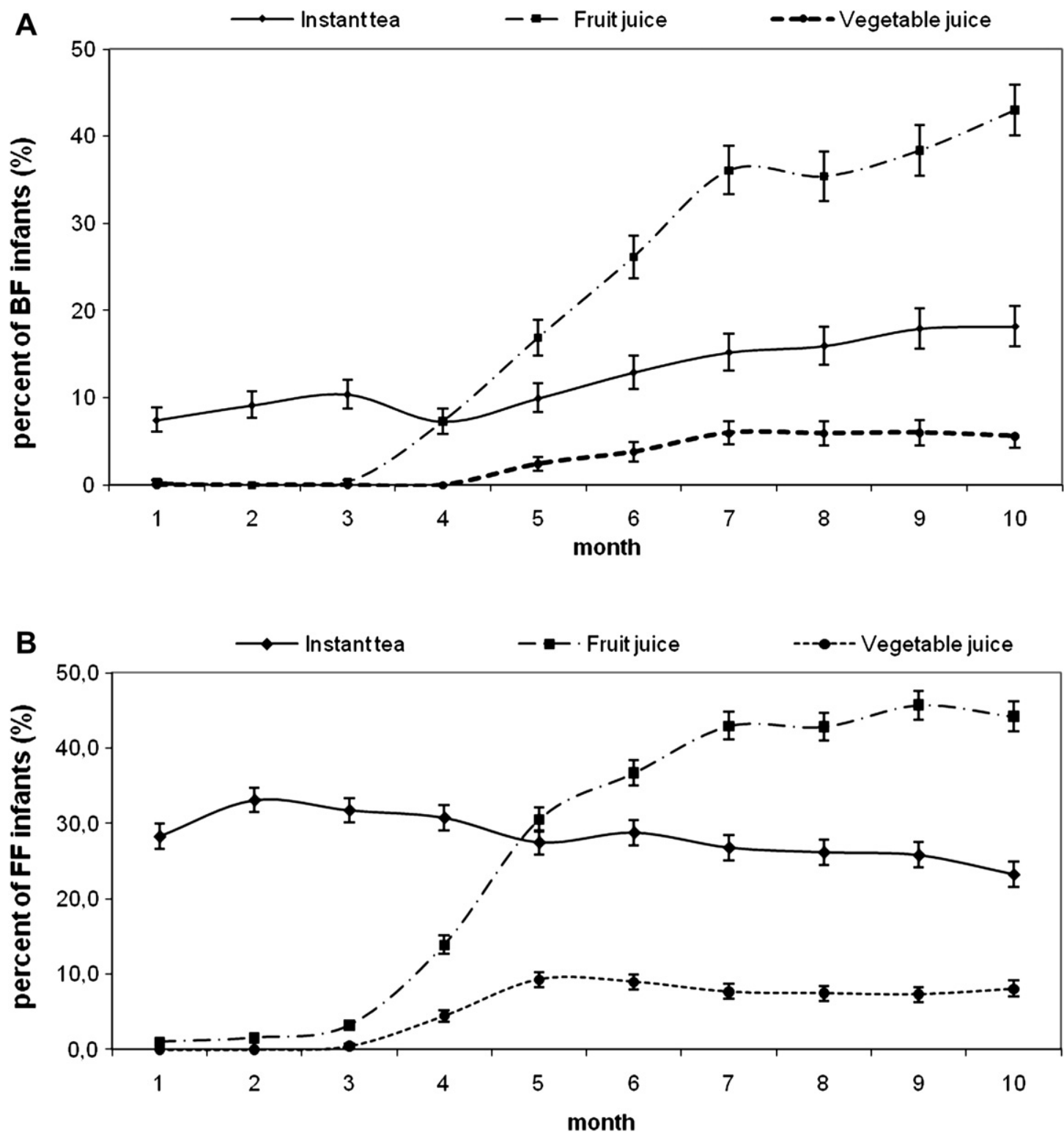

Fig. 1. Percentage (\%) and standard error (SE) of breastfed (A) and formula fed infants (B) with intake of instant tea, fruit juice or vegetable juice during the first year of life. 


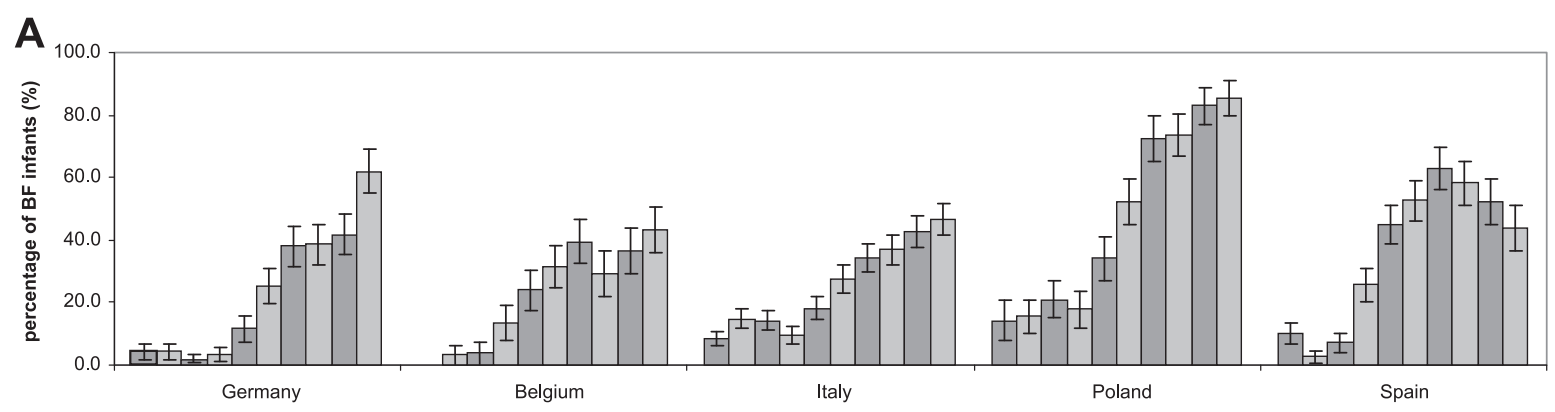

The proportion of breastfed infants with consumption of EPL differed significantly between the countries at the age of 2 to 12 completed months (all $p<0.01$ )

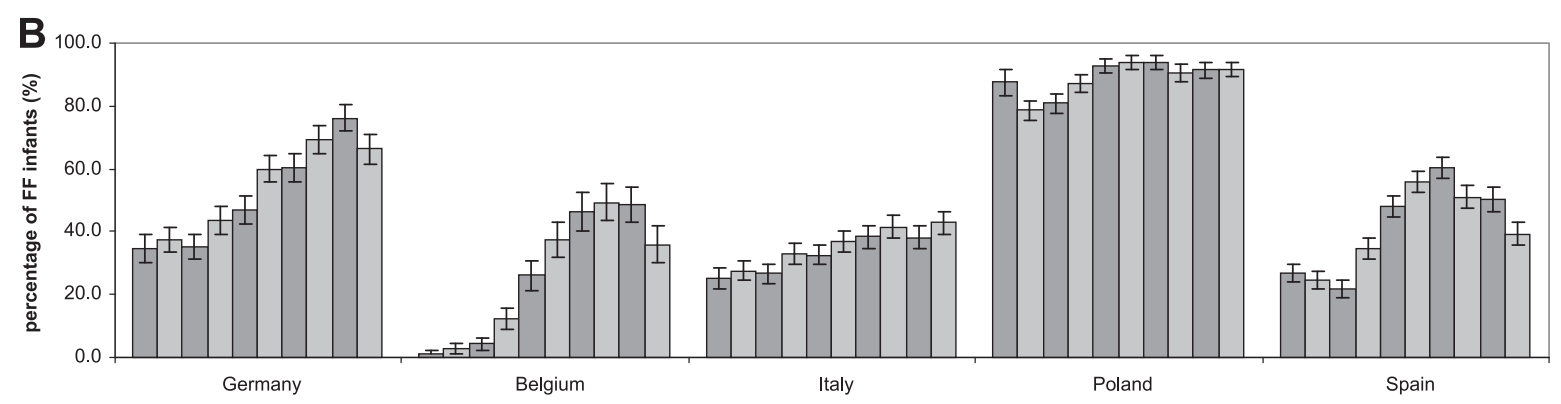

The proportion of formula fed infants with consumption of EPL differed significantly between the countries at the age of 1 to 12 completed months (in all months $p<0.001$ )

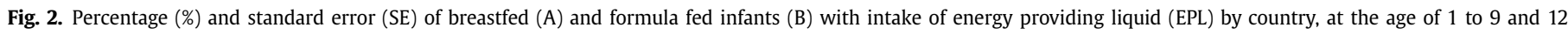
completed months.

Applying a multiple logistic regression for each month on the introduction of EPL in BF infants during the first 7 months of life, including the effects of maternal age, education level, smoking behaviour and country of residence, the main predicting factor for introduction of EPL was consistently the country of residence (Table 1). Polish BF infants had a 17 -fold higher odds to consume EPL at the age of 3 months than BF infants in Germany. Also, in Italy, we found a 10 -fold higher risk for BF infants to receive EPL at the age of 3 months, and in Spain at the age of 4 months, as compared to BF infants in Germany.

We repeated the same analytical approach for instant tea, fruit and vegetable juice. In addition to the effect of country of residence, a low maternal educational level was associated with introduction of EPL and instant tea at the age of 3 completed months (OR 4.3, CI: 1.3-14.0, $p=0.17$ ), and maternal smoking was associated with introduction of fruit juice at 4 (OR 3.2, CI: $1.0-9.8, p=0.04)$ and 6 completed months (OR 2.2, CI: $1.1-4.3, p=0.02$ ) (data not shown).

\section{FF infants}

During the first year of life, $86 \%$ of FF infants received EPL and the median age at introduction was 17 weeks. Some $30.0 \%$ of FF infants received EPL at the age of one month and $43 \%$ and $57 \%$ at the ages of 4 and 6 completed months, respectively. During the first 4 months, instant tea, with up to $33 \%$ of the infants, was the predominant EPL consumed by the FF infants (Fig. 1B). Fruit juices (mainly apple and orange juice) were given to $31 \%$ of FF infants at 5 months and up to $46 \%$ during the following months. Some $5 \%$ of all the $\mathrm{FF}$ infants consumed vegetable juice (mainly carrot juice or mixtures of carrot juice with fruit juice) at 4 months, and less than $10 \%$ during the rest of the year. Other sugared beverages were hardly consumed by FF infants at all $(<0.5 \%)$.

We found significant differences between the countries in EPL consumption (Fig. 2B). During the first 4 months of life, infants in Poland had the highest proportion receiving EPL (79\% to $88 \%$ ), followed by Germany (35\% to $44 \%$ ), Italy (25\% to $33 \%$ ), Spain ( $22 \%$ to $35 \%$ ) and Belgium (1\% to $12 \%$ ).

Instant teas were consumed by $88 \%$ of FF infants in Poland at the age of one month, and between $70 \%$ and $88 \%$ during the first year of life (Table S2). During the first 4 months of life, $28 \%$ to $32 \%$ of FF infants in Germany, $25 \%$ to $31 \%$ in Italy and $26 \%$ to $13 \%$ in Spain consumed some instant tea. In Spain, this proportion declined over the year and, in Belgium, less than $5 \%$ of all FF infants received instant tea.

At the age of 4 months $14 \%$ of FF infants consumed some fruit juice. Italy ( $0 \%$ to $29 \%$ ) had the lowest proportion of infants getting fruit juice during the first year, whereas infants in Germany (2\% to $3 \%$ ) and Spain ( $2 \%$ to $8 \%$ ) already received fruit juice during the first 3 months of life (Table S2). At the age of 4 months (17 weeks), 10\% FF infants in Germany, $11 \%$ in Belgium, 20\% in Poland and 24\% in Spain consumed some fruit juice. In Italy, only $2 \%$ of the FF infants consumed fruit juice at 4 months and $10 \%$ at 6 months, as compared to $39 \%-54 \%$ in the other countries.

Vegetable juice was consumed by Polish and German FF infants between the ages of 3 and 12 completed months, but hardly in other countries (Table S2). Applying a multiple logistic regression model for each of the first 7 months, including maternal age, education level, smoking behaviour and country of residence as covariates for the introduction of EPL or instant tea in FF infants during the first year of life, country of residence was the only consistent predictive factor for an earlier introduction of EPL (in all months, $p<0.001$, Table 1 ). Compared to Germany, FF infants in Poland had an approximately 6 to 14 -fold higher risk for EPL intake. Repeating the analysis only with the introduction of fruit juice in FF infants, the country of residence was also seen to be a risk factor at the ages of 3 to 12 months $(p<0.05)$. Intakes of any EPL were not associated with protein contents of study formula, birth weight or birth order. The two formula groups also did not differ regarding the consumption of fruit juice, vegetable juice or instant tea, with the only exception being a significantly higher fruit juice intake in the high protein group at the age of 5 months $(p=0.026)$. 
Table 1

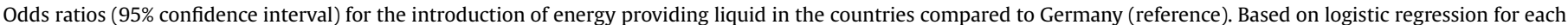
age group (age of 1 to 7 completed months) including maternal age, maternal education level and maternal smoking behaviour.

\begin{tabular}{|c|c|c|c|c|c|c|c|c|c|c|c|c|}
\hline \multirow[t]{2}{*}{ Age (completed months) } & \multicolumn{3}{|c|}{ Belgium } & \multicolumn{3}{|l|}{ Italy } & \multicolumn{3}{|c|}{ Poland } & \multicolumn{3}{|l|}{ Spain } \\
\hline & OR & $95 \% \mathrm{CI}$ & $p$-value & OR & $95 \% \mathrm{CI}$ & $p$-value & OR & $95 \% \mathrm{CI}$ & $p$-value & OR & $95 \% \mathrm{CI}$ & $p$-value \\
\hline \multicolumn{13}{|l|}{ Breastfed infants } \\
\hline \multicolumn{13}{|l|}{ 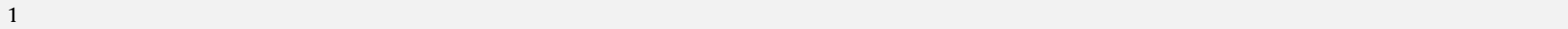 } \\
\hline 2 & & & & 3.92 & $(1.08-14.17)$ & 0.04 & & & & & & \\
\hline 3 & & & & 9.94 & $(1.26-78.51)$ & 0.03 & 17.71 & $(2.06-152.62)$ & 0.01 & & & \\
\hline 4 & & & & & & & 8.20 & $(1.57-42.85)$ & 0.01 & 10.20 & $(2.23-46.70)$ & 0.003 \\
\hline 5 & & & & & & & 3.46 & $(1.14-9.84)$ & 0.02 & 6.38 & $(2.49-16.38)$ & $<0.001$ \\
\hline 6 & & & & & & & 3.40 & $(1.40-8.28)$ & 0.01 & 3.13 & $(1.41-6.95)$ & 0.01 \\
\hline 7 & & & & & & & 3.53 & $(1.40-8.85)$ & 0.01 & 2.74 & $(1.26-5.99)$ & 0.01 \\
\hline \multicolumn{13}{|l|}{ Formula fed infants } \\
\hline 1 & 0.02 & $(0.003-0.15)$ & $<0.001$ & & & & 11.77 & $(5.03-27.54)$ & $<0.001$ & & & \\
\hline 2 & 0.05 & $(0.16-0.18)$ & $<0.001$ & & & & 5.71 & $(3.36-9.70)$ & $<0.001$ & & & \\
\hline 3 & 0.09 & $(0.03-0.25)$ & $<0.001$ & & & & 7.31 & $(4.22-12.68)$ & $<0.001$ & 0.53 & $(0.33-0.85)$ & 0.01 \\
\hline 4 & 0.20 & $(0.10-0.41)$ & $<0.001$ & & & & 8.30 & $(4.54-15.17)$ & $<0.001$ & & & \\
\hline 5 & 0.44 & $(0.24-0.80)$ & 0.01 & 0.58 & $(0.36-0.93)$ & 0.03 & 14.13 & $(6.85-29.16)$ & $<0.001$ & & & \\
\hline 6 & 0.40 & $(0.22-0.72)$ & 0.01 & 0.42 & $(0.26-0.67)$ & $<0.001$ & 9.24 & $(4.24-20.12)$ & $<0.001$ & & & \\
\hline 7 & & & & 0.43 & $(0.26-0.69)$ & $<0.001$ & 9.06 & $(3.97-20.66)$ & $<0.001$ & & & \\
\hline
\end{tabular}

Applying a multiple regression for the first 4 months of life including the effect of country of residence, maternal age and educational level as well as the maternal smoking behaviour, we found that FF infants were 5 times more likely to receive EPL up to 4 months of age (OR:5.1; 95\%CI: 4.1-6.3).

\section{Energy providing liquid and energy intake}

Table 2 shows the total energy intake ( $\mathrm{kcal} / \mathrm{d}$ ), the energy intake from EPL, formula milk and solids of FF infants with and without EPL consumption. FF infants receiving EPL had a significantly lower energy supply through infant formula at the ages of 2 to 5 months. This is also seen in Table S3 where the median intake of EPL $(\mathrm{ml} / \mathrm{d})$, as well as the intake of infant formula $(\mathrm{ml} / \mathrm{d})$ in FF infants with and without EPL, are shown. At the ages of 4 and 5 months, infants receiving EPL consumed a significantly higher energy intake from solids than infants without EPL consumption. But infants with EPL consumption had a significantly lower energy intake from solids at the ages of 7 to 9 and at 12 completed months.

\section{Discussion}

This study shows that EPL are given to a very high proportion of European infants during the first months of life. Some $13 \%$ of BF infants and $43 \%$ of FF infants receive EPL by the age of 4 months, even though such a practice is not supported at all by current recommendations. ${ }^{2}$ There is no nutritional benefit of feeding EPL or fruit juices to infants during the first months of life, but there is a possible risk of displacing nutrient intakes from breast milk or infant formula. ${ }^{15-17}$ Our data analysis revealed a lower formula milk intake (kcal/d) in FF infants who consumed EPL during the first months of life. Furthermore, infants who consumed EPL had a lower solids intake ( $\mathrm{kcal} / \mathrm{d}$ ) during the second half of the first year. The significantly lower infant formula intake in infants with EPL coincides with the time of the highest percentage of infants with instant tea intake as EPL. This is of concern, since instant tea provides only rapidly absorbable carbohydrates and none of the essential nutrients supplied with breast milk or infant formula.

Infants who received EPL had significantly higher energy intakes from solids at the ages of 4 and 5 months as compared to infants who did not receive EPL, suggesting that the provision of EPL is associated with an earlier solid introduction in the infant's diet.
There are markedly different feeding practices between the countries. Both in FF and BF infants, country of residence is a consistent predictive factor for the time of EPL introduction (Table 1). These country differences might be due to differences in local traditions of infant feeding or in communication to parents, but are not explained by infant feeding recommendations which are similar throughout these five countries.

Our findings also show that FF infants receive EPL and solids earlier and to a higher proportion then BF infants, although the recommendations do not differ. ${ }^{18,19}$ Up to the age of 4 months, FF infants were 5 times more likely to receive EPL than BF infants. Parada et al. also found higher consumption frequencies of tea and juices among bottle fed than among breastfed infants in Brazil during the first months of life. ${ }^{20}$ Similarly, Emmett et al. found a higher percentage of British FF infants than BF infants receiving additional drinks other than breast milk or infant formula, and they speculated that this could be due to FF infants being accustomed to drinking from bottles. ${ }^{21}$

In our study group, instant teas were the predominate form of EPL during the first months of life, whereas fruit and vegetable juices were increasingly introduced from 3 to 4 completed months onwards and consumed in higher proportions during the second half of the first year (Figs. 1 and 2). Instant teas might be used in young infants with excessive crying, based on the assumption that infants should get some liquid in addition to breast milk or formula, whereas older infants might get fruit juice as a nutritional supplement or because it may be perceived to be more similar to complementary foods given at older ages. Parada et al. found higher percentages of Brazilian infants with an intake of teas from birth to the age of 4 months ( $29 \%$ for BF infants and $47 \%$ for FF infants) compared to our infants, and even up to $69 \%$ of the infants with an intake of fruit juices during the second half of the first year. ${ }^{20}$ In the Feeding Infants and Toddlers Study (FITS), Menella et al. analysed the foods fed to Hispanic and non-Hispanic infants in the United States, and found similar percentages of infants with fruit juice intake as we found for our FF infants, but a much higher proportion of infants who consumed vegetable juice, even compared to our highest percentage of infants with an intake of vegetable juice who were found in Poland. ${ }^{22}$

Sweet drinks are not recommended in the infant diet because they contribute few nutrients other than energy, whereas appropriate intake of breast milk or infant formula as well as complementary foods should provide the infants with an adequate nutrient 
Table 2

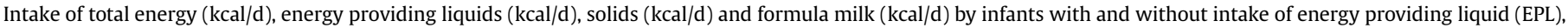
presented as median and interquartile range (IQR).

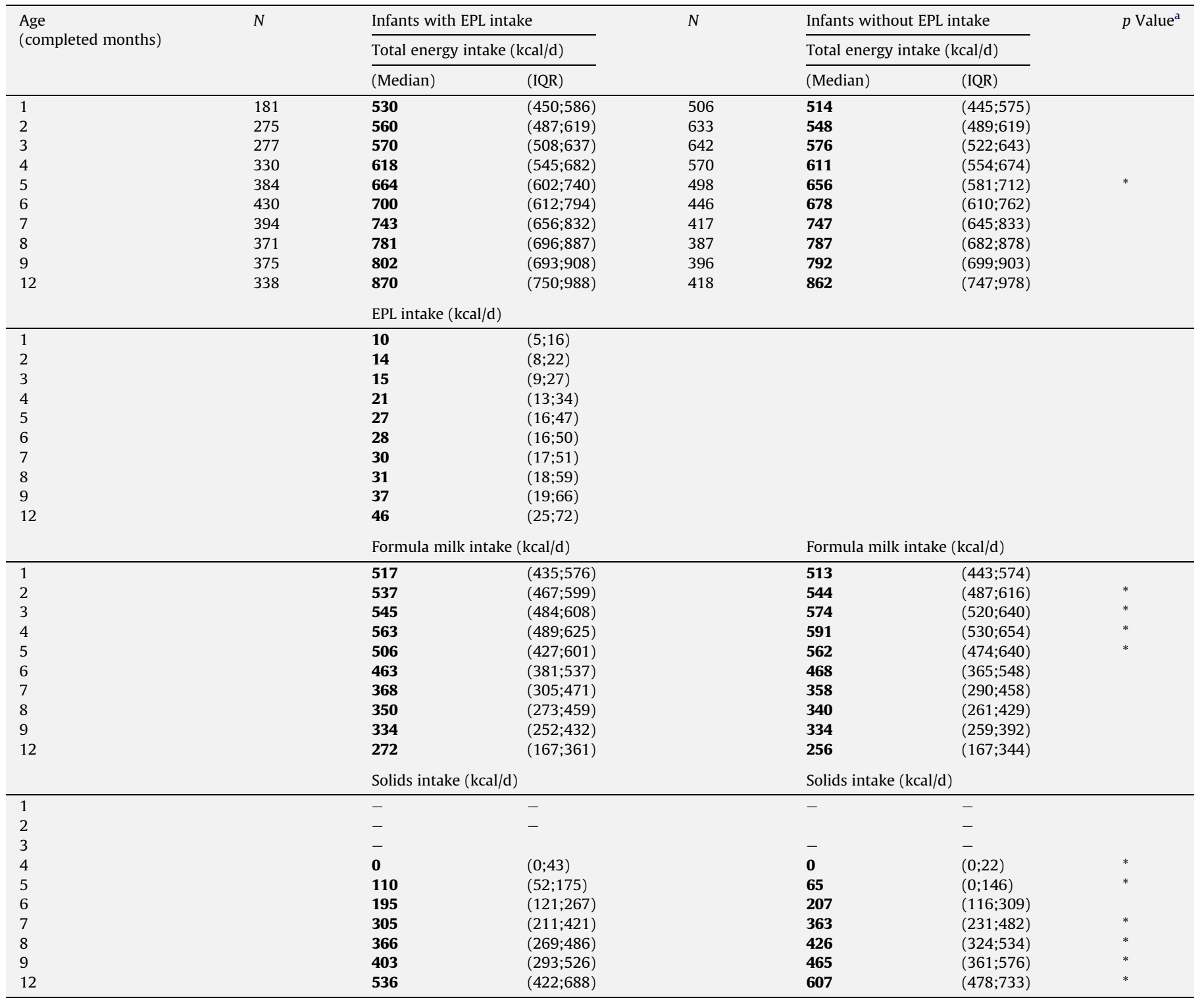

${ }^{\text {a }}$ Comparison was done with the Wilcoxon rank-sum test $\left({ }^{*} p<0.05\right)$.

intake. ${ }^{2,23-26}$ Educational level of the caregiver, affordability of products, consumption habits of parents, guidance by health care professionals, as well as product advertising and marketing all may influence the consumption habits of sweet drinks. ${ }^{27,28,15,16}$

In conclusion, this study shows that, in contrast to current recommendations, EPL are provided surprisingly early and at a surprisingly high rate to infants during the first year of life, particularly in FF infants. The provision of EPL is associated with a lower energy intake from infant formula, earlier introduction of solids, and less energy intake from solids during the second half of the first year of life. Infant feeding practices should be improved by informing health care professionals, parents, and manufacturers of infant food products.

\section{Conflict of interest}

None of the authors has declared a conflict of interest. The project was granted by the Ethical Committee of the Bavarian Board of physicians.

\section{Acknowledgements}

We thank the families who participated in this study, and the collaborating physicians, midwives and nurses for their help in informing and recruiting families. We are very grateful to the study partners for their efforts, endurance and dedication to this work. The European Childhood Obesity Project is carried out with financial support of the European Community, under the 5th Framework Programme for Research, Technology \& Demonstration "Quality of Life and Management of Living Resources", Key Action 1 (Food, Nutrition \& Health), contract number QLK1-CT2002-389 and 6th Framework priority 5.4.3.1 Food quality and safety (Early nutrition programming - long-term follow up of efficacy and safety trials and integrated epidemiological, genetic, animal, consumer and economic research, EARNEST, Food-CT-2005-007036). It does not necessarily reflect the views of the Commission and in no way anticipates its future policy in this area. Berthold Koletzko is the recipient of a Freedom to Discover Award of the Bristol Myers 
Squibb Foundation, New York, NY, USA. The presented data are part of the PhD Thesis in Human Biology submitted by Sonia Schiess to the Medical Faculty, Ludwig-Maximilians-University of Munich.

Statement of authorship: SSch was involved in enrolment of subjects, acquisition and introduction of data, data analysis and interpretation, manuscript writing. VG data management and data analysis, contributed to manuscript writing. SSc participated in study design and in conducting the study, contributed to manuscript writing. VL, FM, AS, FV were involved in enrolment of subjects, in conducting the study, acquisition and introduction of data, and contributed to manuscript writing. BK, principal investigator of the study, contributed to manuscript writing. All authors read and approved the final version of the article.

Names for Potential Reviewers

Cristina Campoy, Granada, Spain, ccampoy@ugr.es

Kim F Michaelsen, Copenhagen, Denmark, kfm@kvl.dk

Olle Hernell, Umea, Sweden, olle.hernell@pediatri.umu.se

\section{Appendix. Supplementary data}

Supplementary data associated with this article can be found in the online version, at doi:10.1016/j.clnu.2010.04.003.

\section{References}

1. ESPGHAN Committee on Nutrition, Agostoni C, Braegger C, Decsi T, Kolacek $S$ Koletzko B, Michaelsen KF, et al. Breast-feeding: a Commentary by the ESPGHAN Committee on Nutrition. J Pediatr Gastroenterol Nutr 2009;49: $112-25$.

2. ESPGHAN Committee of Nutrition, Agostoni C, Decsi T, Fewtrell M, Goulet O, Kolacek S, Koletzko B, et al. Complementary feeding: a commentary by the ESPGHAN Committee on Nutrition. J Pediatr Gastroenterol Nutr 2008;46:99-110.

3. Kersting M. Ernährung des gesunden Säuglings. Monatsschrift Kinderheilkunde 2001;149:4-10.

4. Committee on Nutrition. The use and misuse of fruit juice in pediatrics. Pediatrics 2001;107:1210-3.

5. Ludwig DS, Peterson KE, Gortmaker SL. Relation between consumption of sugar-sweetened drinks and childhood obesity: a prospective, observational analysis. Lancet 2001;357:505-8.

6. Malik VS, Schulze MB, Hu FB. Intake of sugar-sweetened beverages and weight gain: a systematic review. Am J Clin Nutr 2006;84:274-88.

7. Vartanian LR, Schwartz MB, Brownell KD. Effects of soft drink consumption on nutrition and health: a systematic review and meta-analysis. Am J Public Health 2007;97(4):667-75

8. Lifschitz $\mathrm{CH}$. Carbohydrate absorption from fruit juices in infants. Pediatrics 2000;105:e4
9. Cole CR, Rising R, Lifshitz F. Consequences of incomplete carbohydrate absorption from fruit juice consumption in infants. Arch Pediatr Adolesc Med 1999;153:1098-102.

10. Smith MM, Davis M, Chasalow FI, Lifshitz F. Carbohydrate absorption from fruit juice in young children. Pediatrics 1995;95:340-4

11. van der Merwe J, Kluyts M, Bowley N, Marais D. Optimizing the introduction of complementary foods in the infant's diet: a unique challenge in developing countries. Matern Child Nutr 2007;3:259-70.

12. Fomon SJ. Infant feeding in the 20th century: formula and Beikost. J Nutr 2001;131:409S-4420.

13. Koletzko B, von Kries R, Closa R, Escribano J, Scaglioni S, Giovannini M, et al. Can infant feeding choices modulate later obesity risk? Am J Clin Nutr 2009;89 (5):S1502-S1508.

14. Koletzko B, von Kries R, Closa R, Escribano J, Scaglioni S, Giovannini M, et al. Lower protein in infant formula is associated with lower weight up to age $2 \mathrm{y}$ : a randomized clinical trial. Am J Clin Nutr 2009;89:1-10.

15. Marshall TLS, Broffitt B, Eichenberger-Gilmore J, Stumbo J. Beverage consumption and infant nutrition - benefits of milk vs. juice. Nutr Res Newslett; 2003.

16. Saalfield S, Jackson-Allen P. Biopsychosocial consequences of sweetened drink consumption in children $0-6$ years of age. Pediatr Nurs 2006;32(5):460-71.

17. Gibson SA. Non-milk extrinsic sugars in the diets of pre-school children: association with intakes of micronutrients, energy, fat and NSP. Br J Nutr 1997; 78:367-78.

18. Fewtrell MS, Morgan JB, Duggan C, Gunnlaugsson G, Hibberd P, Lucas A, et al. Optimal duration of exclusive breastfeeding: what is the evidence to support current recommendations? Am J Clin Nutr 2007;85:635S-638.

19. Schiess S, Grote V, Scaglioni S, Luque V, Martin F, Stolarczyk A, et-al. Introduction of complementary feeding in five European countries. J Pediatr Gastroenterol Nutr 49, 1-8.

20. Parada C, Carvalhaes MABL, Jamas MT. Complementary feeding practices to children during their first year of life. Rev Latino-am Enfermagem 2007;15 (2):282-9.

21. Emmett P, North K, Noble S, ALSPAC Study Team. Types of drinks consumed by infants at 4 and 8 months of age: a descriptive study. Pub Health Nutr 3(2), 211-217.

22. Mennella J, Ziegler P, Briefel R, Novak T. Feeding infants and toddlers study: the types of foods fed to Hispanic infants and toddlers. J Am Diet Assoc 2006;106: S96-S106.

23. Daelmans B, Martines J, Saadeh R. Conclusions of the global consultation on complementary feeding. Food Nutr Bull 2003;24(1):126-9.

24. Dewey, K.G., Lutter, Chessa, Martines, J., Daelmans, B., and WHO Global Consultation on Complementary Feeding. Guiding principles for complementary feeding of the breastfed child. 1-37.

25. Monte CMG, Giugliani ERJ. Recommendations for the complementary feeding of the breastfed child. J Pediatr (Rio J) 2004;80(5 Suppl.):S131-S141.

26. WHO. Complementary feeding. $1-24$.

27. Fisher JO, Mitchell DC, Smiciklas-Wright H, Birch LL. Maternal milk consumption predicts the tradeoff between milk and soft drinks in young girls' diets. J Nutr 2001;131:246-50.

28. Hendricks K, Briefel R, Novak T, Ziegler P. Maternal and Child characteristics associated with infant and toddler feeding practices. I Am Diet Assoc 2006;106:135-48. 